\title{
Involvement of ELAV RNA-binding proteins in the post-transcriptional regulation of $\mathrm{HO}-1$
}

\author{
Marialaura Amadio ${ }^{1+}$, Giovanni Scapagnini ${ }^{2,3}{ }^{*}{ }^{\dagger}$, Sergio Davinelli $^{2}$, Vittorio Calabrese ${ }^{3,4}$, Stefano Govoni ${ }^{1}$ \\ and Alessia Pascale ${ }^{1}$
}

${ }^{1}$ Department of Drug Sciences, Section of Pharmacology, University of Pavia, Pavia, Italy

${ }^{2}$ Department of Medicine and Health Sciences, University of Molise, Campobasso, Italy

${ }^{3}$ Inter-University Consortium "SannioTech", Benevento, Italy

${ }^{4}$ Department of Biomedical Sciences, University of Catania, Catania, Italy

\section{Edited by:}

Rosanna Parlato, Ulm University, Germany

Reviewed by:

Zsolt Radak, Semmelwies

University, Hungary

Pasquale Vito, Università del

Sannio, Italy

\section{${ }^{*}$ Correspondence:}

Giovanni Scapagnini, Department of

Medicine and Health Sciences,

University of Molise, Via De Sanctis

86100, Campobasso, Italy

e-mail: g.scapagnini@gmail.com

${ }^{\dagger}$ These authors have contributed

equally to this work.
Heme oxygenase-1 ( $\mathrm{HO}-1)$ is an inducible rate-controlling enzyme of heme catabolism. The cytoprotective function of HO-1 activity has been verified in multiple studies, and together with its by-products is considered a key component of the cellular stress response. The transcriptional induction of $\mathrm{HO}-1$ has been largely studied in response to multiple forms of stressful stimuli but our understanding of HO-1 post-transcriptional control mechanisms in neuronal cells is currently lacking. In the present report we show the involvement of the RNA-binding proteins (RBPs) embryonic lethal abnormal vision (ELAV) in the regulation of HO-1 gene expression. Our study demonstrates a specific binding between HO-1 messenger RNA (mRNA) and ELAV proteins, accompanied by an increased expression of HO-1 at protein level, in a human neuroblastoma cell line treated with hemin. Clarifying the induction of HO-1 expression at post-transcriptional level may open therapeutic perspectives for treatments associated with the modulation of HO-1 expression.

Keywords: heme oxygenase-1, ELAV, hemin, post-transcriptional regulation

\section{INTRODUCTION}

Heme oxygenase-1 (HO-1) is an intracellular enzyme that catalyzes the initial and rate-limiting step in the oxidative degradation of heme, and generates biliverdin, free iron $\left(\mathrm{Fe}^{2+}\right)$, and carbon monoxide (CO; Calabrese et al., 2006). HO-1 is a heat shock protein (HSP32) and it is recognized as one of the major stress-inducible protein in mammalian cells (Maines, 1997). HO-1 activity can increase several-fold in response to a wide variety of stimuli that cause changes in the cellular redox state (Ryter and Choi, 2002). The products of heme metabolism such as bilirubin, ferritin and $\mathrm{CO}$, mediate many of the anti-inflammatory and antioxidant effects associated with the potent cytoprotection provided by $\mathrm{HO}-1$ enzymatic system (Kirkby and Adin, 2006). HO-1 expression is induced ubiquitously in response to oxidative challenges but brain tissue is particularly susceptible to free radical damage (Poon et al., 2004). Indeed, there is an increasing support that HO-1 plays a crucial protective role in the central nervous system (CNS), primarily in astrocytes and microglia/macrophages, particularly during aging and in several disease states where oxidative stress is implicated, including Alzheimer's disease (AD), Parkinson's disease (PD) and Huntington's disease (HD; Calabrese et al., 2004). Although numerous published reports have demonstrated multiple beneficial effects of HO-1, its mechanism of action has not been completely elucidated. Expression of HO-1 is regulated essentially at transcriptional level, even though a posttranscriptional modulation of HO-1 messenger RNA (mRNA) in distinct cellular contexts has been described (Gozzelino et al.,
2010). For instance, in human dermal fibroblasts hypoxia regulates $\mathrm{HO}-1$ gene expression by a specific post-transcriptional mechanism: stabilization of mRNA (Kitamuro et al., 2003). Therefore, the regulation of HO-1 mRNA levels in response to cellular stress may be induced by both transcriptional and post-transcriptional events that act independently, and vary in function of the stress inducer (Leautaud and Demple, 2007). Although post-transcriptional control mechanisms are not yet fully understood, it is clear that they are key determinants in the regulation of mRNA stability and translation (Bolognani and Perrone-Bizzozero, 2008; Pascale et al., 2008; Keene, 2010). In particular, RNA-binding proteins (RBPs) regulate gene expression at post-transcriptional level and influence pre-mRNA processing as well as transport, localization, stability and translation of target mRNAs (Dreyfuss et al., 2002). RBPs are crucial in many aspects of cellular physiology and may play a direct role in the pathophysiology of neurodegenerative diseases (Pascale and Govoni, 2012; Perrone-Bizzozero and Bird, 2013; Romano and Buratti, 2013). Recent studies have identified hundreds of RBPs previously unknown (Castello et al., 2012) and a new and fascinating idea is that neurons have their own systems for regulating RNA metabolism, processing, localization, and expression (Darnell, 2013). In this context, embryonic lethal abnormal vision (ELAV) proteins are RBPs mostly expressed in neurons and post-transcriptional regulation in neuronal cells strongly depends on the control exerted by these RBPs (Pascale et al., 2008). In vertebrates, the ELAV (or $\mathrm{Hu}$ ) family comprises the neuronspecific members $\mathrm{HuB}, \mathrm{HuC}$ and $\mathrm{HuD}$, and the ubiquitously 
expressed HuR (Colombrita et al., 2013). Moreover, emerging insights into the regulation by which cells establish patterns of gene expression suggest that post-transcriptional events influence inducible genes and signaling cascades such as HSPs and oxidative stress-activated pathways (Abdelmohsen et al., 2008; Amadio et al., 2008). HO-1 gene product and its mRNA abundance is controlled at various stages and it was reported that HO-1 induction by nitric oxide (NO) is regulated by the HuR/ELAV. Interestingly, the HuR/ELAV was found to associate with $\mathrm{HO}-1$ mRNA, and this interaction increased following $\mathrm{NO}$ treatment (Kuwano et al., 2009). The HO-1 chemical inducer hemin is a highly reactive compound exhibiting pro-oxidant properties in several biochemical reactions. In the present study, we investigated, in SH-SY5Y human neuroblastoma cells, the expression of HO-1 mRNA and protein following hemin exposure and whether in these conditions HO-1 mRNA may represent a target of ELAV RBPs.

\section{MATERIALS AND METHODS CELL CULTURES AND TREATMENTS}

The SH-SY5Y human neuroblastoma cells were grown in Eagle's minimum essential medium supplemented with $10 \%$ fetal calf serum, $1 \%$ penicillin-streptomycin, L-glutamine (2 mM), nonessential amino acids $(1 \mathrm{mM})$, and sodium pyruvate $(1 \mathrm{mM})$ at $37^{\circ} \mathrm{C}$ in an atmosphere of $5 \% \mathrm{CO}_{2}$ and $95 \%$ humidity, as previously described (Racchi et al., 2003). The cells were exposed to the solvent (PBS) or to hemin (50 or $100 \mu \mathrm{M}$ as reported in figure legends) for $2 \mathrm{~h}$.

\section{REVERSE-TRANSCRIPTION AND REAL-TIME QUANTITATIVE POLYMERASE CHAIN REACTION (qPCR)}

Total RNA was extracted from cells using the RNeasy Micro Kit (Qiagen, Milan, Italy). The reverse transcription was performed following standard procedures. PCR amplifications were performed using the LightCycler instrument (Roche Molecular Biochemicals) in the presence of QuantiTect SYBR Green PCR mix (Qiagen, Milan, Italy), with primers designed by using the PRIMER3 software. ${ }^{1}$ Primer sequences were as follows: HO-1, 5'-AGC AAC AAA GTG CAA GAT TCT GC-3' (forward); 5' CAG CAT GCC TGC ATT CAC ATG-3' (reverse); product size: 161 bp; RPL6 (Ribosomal Protein L6), 5' - AGA TTA CGG AGC AGC GCA AGA TTG-3' (forward), 5'-GCA AAC ACA GAT CGC AGG TAG CCC-3' (reverse). RPL6 mRNA was chosen as the reference mRNA on which HO-1 was normalized because this RNA remained substantially stable during the treatments. Since RPL6 mRNA does not bear ARE sequences, it was also used as negative control in the immunoprecipitation experiments coupled with real-time quantitative polymerase chain reaction (qPCR).

\section{IMMUNOPRECIPITATION}

Following treatment, SH-SY5Y cells were harvested and homogenized in a buffer [containing $20 \mathrm{mM}$ Tris- $\mathrm{HCl}(\mathrm{pH}$ 7.4), $2 \mathrm{mM}$ EDTA, $0.5 \mathrm{mM}$ EGTA, $50 \mathrm{mM}$ 2-mercaptoethanol, $0.32 \mathrm{mM}$ sucrose, and a protease inhibitor cocktail] by using a teflon/glass homogenizer. Immunoprecipitation was performed

\footnotetext{
${ }^{1}$ http://bioinfo.ut.ee/primer3-0.4.0/primer3/input.htm
}

on total homogenates according to a previously published protocol (Amadio et al., 2009). Briefly, immunoprecipitation was carried out at room temperature for $2 \mathrm{~h}$ using $1 \mu \mathrm{g}$ of anti-ELAV antibody (Santa Cruz Biotech, CA, USA) per $50 \mu \mathrm{g}$ of proteins diluted with an equal volume of $2 \times$ Immunoprecipitation Buffer [2\% Triton X-100, $30 \mathrm{mM} \mathrm{NaCl}, 20 \mathrm{mM}$ Tris-HCl (pH 7.4), $2 \mathrm{mM}$ EDTA, $2 \mathrm{mM}$ EGTA, $0.4 \mathrm{mM}$ sodium vanadate, protease inhibitor cocktail and a RNAase inhibitor] in presence of $50 \mu \mathrm{l}$ of protein A/G plus agarose beads (Santa Cruz Biotech, CA, USA) previously blocked with 5\% BSA in PBS. The samples were finally subjected to RNA extraction and reverse transcription. The negative control was obtained in the same conditions, but in presence of an irrelevant antibody with the same isotype of the specific immunoprecipitating antibody. For binding assay, $100 \mu \mathrm{l}$ of the immunoprecipitation mixes were collected from each sample and used as "input signal" to normalize the realtime qPCR data. For both HO-1 and RPL6, the mRNA content present in the immunoprecipitated pellet has been normalized on the mRNA amount present in the relative "input signal".

\section{WESTERN BLOTTING}

Total lysate were diluted in $2 \times$ sodium dodecyl sulfate (SDS) protein gel loading solution, boiled for $5 \mathrm{~min}$, separated by $12 \%$ SDSpolyacrylamide gel electrophoresis, and then processed following standard procedures. The antibodies anti-ELAV, anti-HO-1, and anti- $\alpha$-tubulin (Santa Cruz Biotechnology, Santa Cruz, CA) were diluted in TBST buffer [ $10 \mathrm{mM}$ Tris- $\mathrm{HCl}, 100 \mathrm{mM} \mathrm{NaCl}, 0.1 \%$ (v/v) Tween 20, pH 7.5] containing 5\% milk. The nitrocellulose membranes were processed with Pierce ECL Plus from Thermo Scientific (Rockford, IL, USA). The experiments were performed at least on three different cell preparations using $\alpha$-tubulin to normalize data. Densitometric analysis was performed using the NIH Image software. ${ }^{2}$

\section{DATA ANALYSIS}

For statistical analysis the GraphPad Instat statistical package (version 3.05 GraphPad software, San Diego, CA, USA) was used. The data were analyzed by analysis of variance (ANOVA) followed, when significant, by an appropriate post hoc comparison test, as indicated in figure legends. Differences were considered statistically significant when $p$ values $\leq 0.05$.

\section{RESULTS}

\section{HEMIN INCREASES H0-1 mRNA LEVELS IN SH-SY5Y CELLS}

To determine whether hemin treatment affects HO-1 expression in human neuroblastoma SH-SY5Y cells, we first measured HO1 mRNA levels in total homogenates of cells treated with hemin $(100 \mu \mathrm{M})$ for $2 \mathrm{~h}+4 \mathrm{~h}$ recovery. Real-time qPCR data demonstrated a marked increase of HO-1 mRNA level in $2 \mathrm{~h}$ hemintreated samples followed by $4 \mathrm{~h}$ recovery as compared to the other samples (Figure 1). RPL6 mRNA level remained substantially stable during the experiments (data not shown). These data reveal that in SH-SY5Y cells hemin treatment up-regulates the expression of HO-1 mRNA.

\footnotetext{
${ }^{2}$ http://rsb.info.nih.gov/nih-image
} 


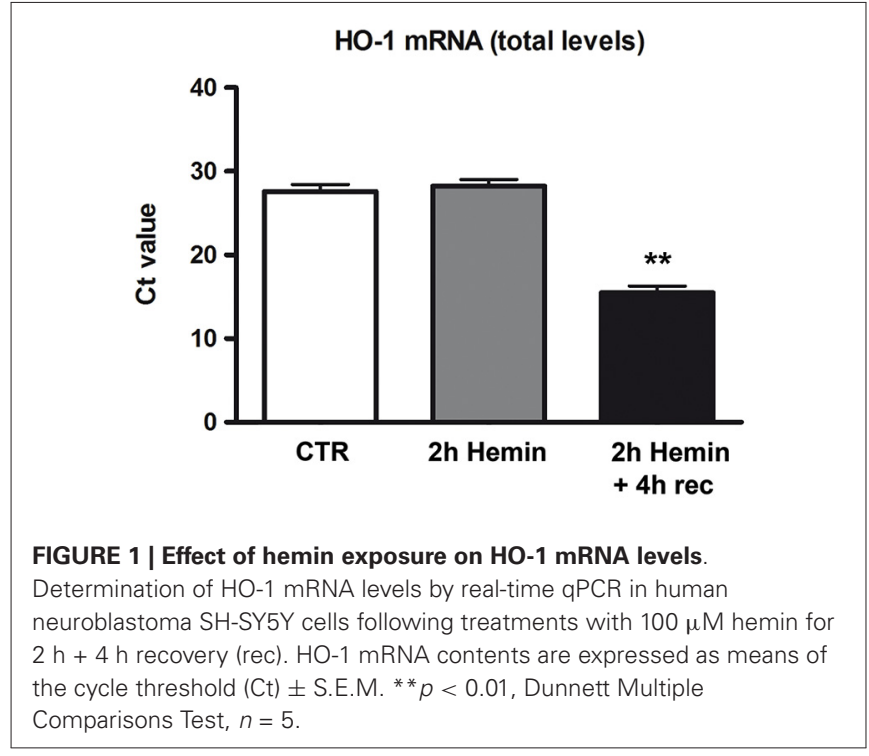

\section{ASSOCIATION OF HO-1 mRNA WITH ELAV RBPS}

To test the hypothesis that ELAV RBPs interact with HO-1 mRNA following hemin treatment, we performed an immunoprecipitation assay on SH-SY5Y cells, which express all the four isoforms of ELAV proteins, followed by real-time qPCR. As shown in Figure 2, the association of ELAV RBPs with HO-1 mRNA occurs; indeed, we found that the amount of HO-1 transcript bound by ELAV proteins in the total homogenate is increased following $2 \mathrm{~h}$ hemin stimulus $+4 \mathrm{~h}$ recovery (Figure 2). Moreover, the housekeeping RPL6 mRNA was almost undetectable in the same immunoprecipitated pellets (Figure 2), confirming the existence of a specific binding between ELAV proteins and $\mathrm{HO}-1$ mRNA.

\section{HEMIN TREATMENT UPREGULATES HO-1 PROTEIN LEVELS IN A CONCENTRATION-DEPENDENT MANNER}

We finally measured HO-1 protein levels by Western blotting, finding an increase after $2 \mathrm{~h}$ hemin exposure followed by $4 \mathrm{~h}$ recovery (Figure 3 ). Interestingly, in basal conditions, HO-1 protein content is almost undetectable and its increase is proportional to hemin concentration, reaching statistical significance only at $100 \mu \mathrm{M}$ concentration (Figure 3). In the same samples we also measured ELAV proteins levels, finding they are not modified following hemin stimulus (not shown).

\section{DISCUSSION}

HO-1 is one of the main players of the endogenous antioxidant responses and since it provides cytoprotection against various neurotoxic insults, it is crucial to elucidate how HO-1 gene expression is regulated. A comprehensive knowledge regarding HO-1 expression in human neural cells is still lacking. Although most studies have focused on the molecular mechanisms responsible for the cytoprotective effects of HO-1, the importance of post-transcriptional processes in the modulation of HO-1 gene expression has been greatly underestimated. Furthermore, there is an emerging consensus that neuronal transcripts are

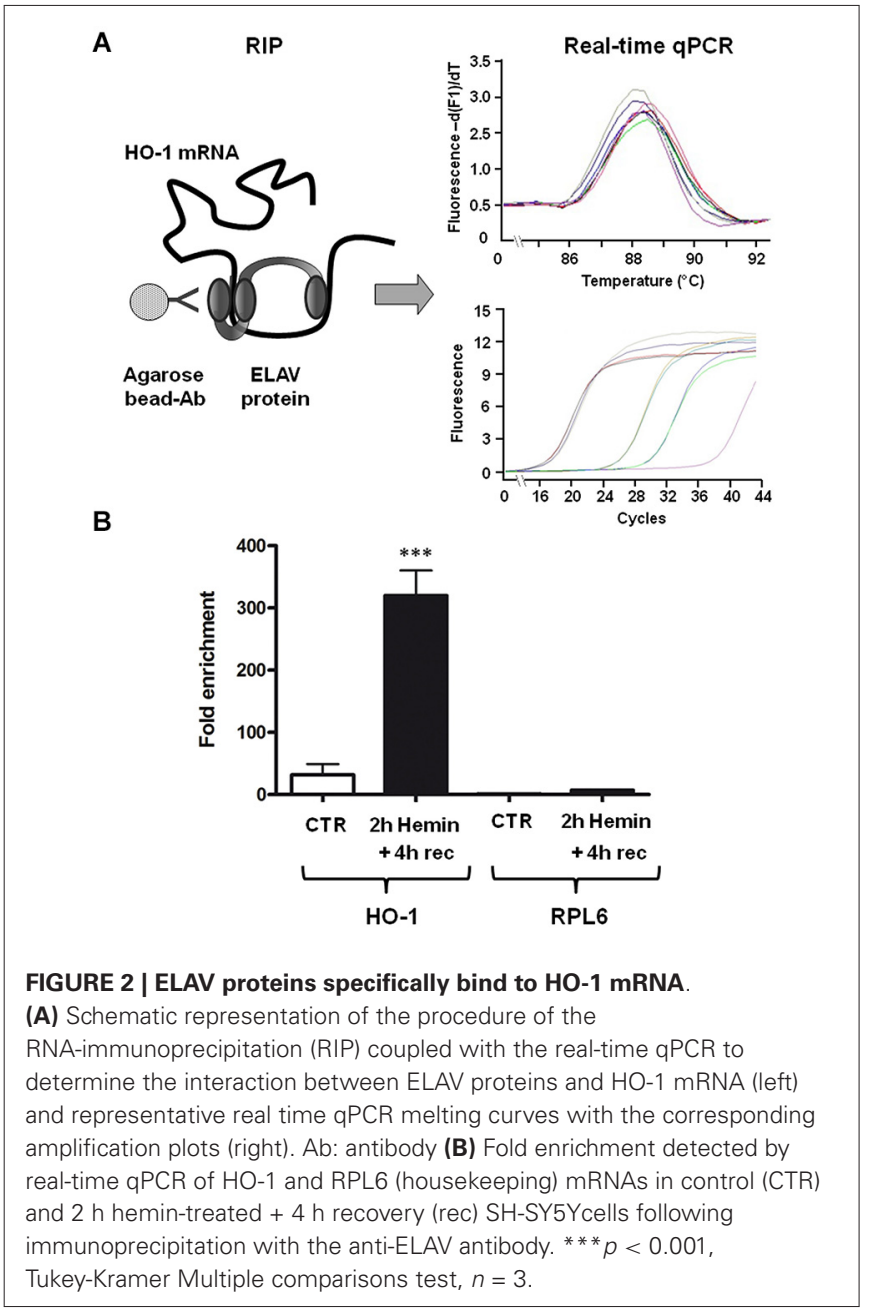

differentially processed in the brain than in other tissues (Darnell, 2013). Interestingly, it was recently demonstrated that RBPs and non-coding RNAs are critical components underlying the post-transcriptional mechanisms for the coordinate regulation of mRNA expression in neuronal systems (Loya et al., 2010). Human ELAV RBPs are involved in the post-transcriptional control of several early-responsive genes such as MYC, FOS and cytokines (Keene, 2007; Papadopoulou et al., 2013). Since HO-1 system is a promising approach to treat specific disorders and its induction has significant consequences in the CNS, the present study explores the role of ELAV proteins in the regulation of $\mathrm{HO}$ 1 expression at both mRNA and protein levels. In particular, we treated human neuroblastoma SH-SY5Y cells with hemin, a pro-oxidant molecule. Although HO-1 is activated by various electrophilic compounds including polyphenols (Davinelli et al., 2013), hemin is one of the most effective stressors in terms of reactive oxygen species (ROS) production and it is widely used to study HO-1 function. Therefore, we chose hemin as a challenge for our experiments. However, it is essential to point out that at elevated concentrations hemin may contribute to cell injury by cytotoxic effects, although strengthening endogenous defense against oxidative stress, at nontoxic concentrations its therapeutic 


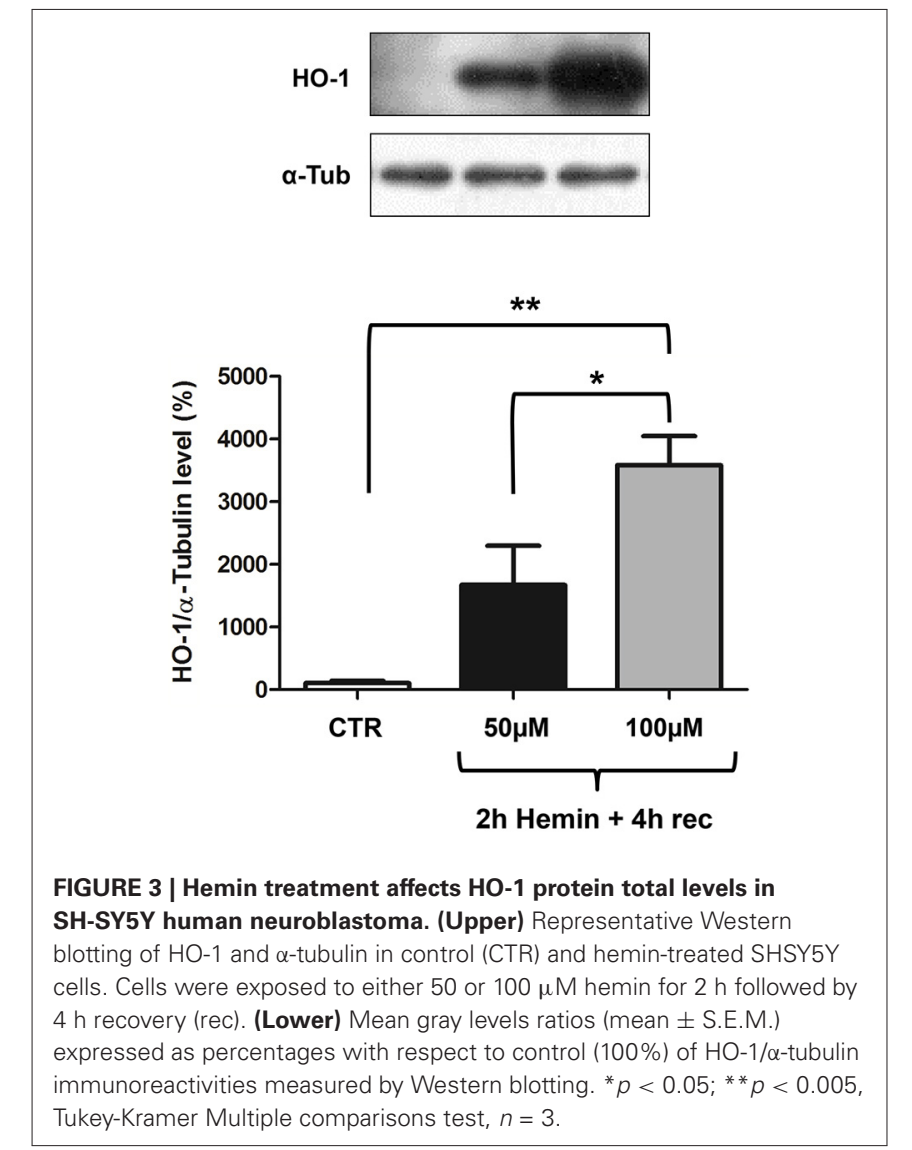

potential has been reported in multiple acute injury models, including those at brain level (Lu et al., 2014). In our study, we found that HO-1 expression is induced in $100 \mu \mathrm{M}$ hemin-treated neuronal cells, as demonstrated by real time qPCR and Western blotting experiments (Figures 1, 3). This finding is consistent with previous studies showing the activation of HO- 1 by hemin in human neuroblastoma cells (Nakaso et al., 2003). Interestingly, and in accordance with previous evidence in rat hippocampal neurons (Scapagnini et al., 2004), we observed that, in human SH-SY5Y neuroblastoma cells, $\mathrm{HO}-1$ protein expression is almost absent in basal conditions and it is strongly induced by hemin in a concentration-dependent manner (Figure 3). However, we cannot exclude that the sensitivity of Western blotting technique is not sufficient to detect very low amount of protein as in control cells. The hemin-induced increase of $\mathrm{HO}-1$ protein may be due to a positive regulation of $\mathrm{HO}-1$ at post-transcriptional level. Indeed, our results shows a specific association between ELAV and HO-1 mRNA (Figure 2), suggesting that this binding may have potential consequences for $\mathrm{HO}-1$ protein expression. Our hypothesis is that HO-1 is present as mRNA in control cells so that, as many early response genes, it can be rapidly translated following a specific stress stimulus, such as hemin exposure, thus contributing to the physiological cellular response.

Although it was well-established that HO-1 is highly inducible by a large number of stressful stimuli such as heme or certain other metalloporphyrins, the regulation of gene expression and
Oxidative stress (i.e. hemin)

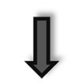

ELAV proteins binding to $\mathrm{HO}-1$ mRNA

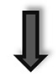

$\uparrow \mathrm{HO}-1$ expression (mRNA and protein)

FIGURE 4 | Flow chart depicting the ELAV/HO-1 cascade, in which an oxidative stress stimulus favors ELAV proteins binding to HO-1 mRNA, producing an increase of HO-1 expression at post-transcriptional level. See the text for more details.

induction of HO-1 is complicated by the fact that its regulatory response is not restricted to heme or other physical and chemical factors. A purpose of this study was to bridge the significant gap in understanding the regulation of $\mathrm{HO}-1$ induction in neural cells. Previous evidence from our group showed that the ELAV RBPs are involved in regulating the post-transcriptional fate of HSP70 and SOD-1 mRNA following $\mathrm{H}_{2} \mathrm{O}_{2}$-mediated oxidative stress in SH-SY5Y cells (Amadio et al., 2008; Milani et al., 2013). HSP70, SOD-1 and HO-1 modulate crucial defensive mechanisms for neurons exposed to an oxidant challenge. Consistently with the data reported for the other genes, the results described in this report suggest a novel role for ELAV RBPs in regulating, post-transcriptionally, $\mathrm{HO}-1$ expression in $\mathrm{SH}-$ SY5Y neuronal cells, as schematically represented in Figure 4. Future studies are needed to identify whether this interaction is a characteristic feature of $\mathrm{HO}-1$ induction during the cellular stress response.

\section{REFERENCES}

Abdelmohsen, K., Kuwano, Y., Kim, H. H., and Gorospe, M. (2008). Posttranscriptional gene regulation by RNA-binding proteins during oxidative stress: implications for cellular senescence. Biol. Chem. 389, 243-255. doi: 10.1515/BC. 2008.022

Amadio, M., Pascale, A., Wang, J., Ho, L., Quattrone, A., Gandy, S., et al. (2009). nELAV proteins alteration in Alzheimer's disease brain: a novel putative target for amyloid-beta reverberating on AbetaPP processing. J. Alzheimers Dis. 16, 409-419. doi: 10.3233/JAD-2009-0967

Amadio, M., Scapagnini, G., Laforenza, U., Intrieri, M., Romeo, L., Govoni, S., et al. (2008). Post-transcriptional regulation of HSP70 expression following oxidative stress in SH-SY5Y cells: the potential involvement of the RNA-binding protein HuR. Curr. Pharm. Des. 14, 2651-2658. doi: 10.2174/13816120878 6264052

Bolognani, F., and Perrone-Bizzozero, N. I. (2008). RNA-protein interactions and control of mRNA stability in neurons. J. Neurosci. Res. 86, 481-489. doi: 10. 1002/jnr.21473

Calabrese, V., Boyd-Kimball, D., Scapagnini, G., and Butterfield, D. A. (2004). Nitric oxide and cellular stress response in brain aging and neurodegenerative disorders: the role of vitagenes. In Vivo 18, 245-267.

Calabrese, V., Butterfield, D. A., Scapagnini, G., Stella, A. M., and Maines, M. D. (2006). Redox regulation of heat shock protein expression by signaling involving nitric oxide and carbon monoxide: relevance to brain aging, neurodegenerative disorders and longevity. Antioxid. Redox Signal. 8, 444-477. doi: 10.1089/ars. 2006.8.444

Castello, A., Fischer, B., Eichelbaum, K., Horos, R., Beckmann, B. M., Strein, C., et al. (2012). Insights into RNA biology from an atlas of mammalian mRNAbinding proteins. Cell 149, 1393-1406. doi: 10.1016/j.cell.2012.04.031 
Colombrita, C., Silani, V., and Ratti, A. (2013). ELAV proteins along evolution: back to the nucleus? Mol. Cell. Neurosci. 56, 447-455. doi: 10.1016/j.mcn.2013. 02.003

Darnell, R. B. (2013). RNA protein interaction in neurons. Annu. Rev. Neurosci. 36, 243-270. doi: 10.1146/annurev-neuro-062912-114322

Davinelli, S., Di Marco, R., Bracale, R., Quattrone, A., Zella, D., and Scapagnini, G. (2013). Synergistic effect of L-Carnosine and EGCG in the prevention of physiological brain aging. Curr. Pharm. Des. 19, 2722-2727. doi: 10. 2174/1381612811319150007

Dreyfuss, G., Kim, V. N., and Kataoka, N. (2002). Messenger-RNA-binding proteins and the messages they carry. Nat. Rev. Mol. Cell Biol. 3, 195-205. doi: 10 $1038 / \mathrm{nrm} 760$

Gozzelino, R., Jeney, V., and Soares, M. P. (2010). Mechanisms of cell protection by heme oxygenase-1. Annu. Rev. Pharmacol. Toxicol. 50, 323-354. doi: 10. 1146/annurev.pharmtox.010909.105600

Keene, J. D. (2007). RNA regulons: coordination of post-transcriptional events. Nat. Rev. Genet. 8, 533-543. doi: 10.1038/nrg2111

Keene, J. D. (2010). Minireview: global regulation and dynamics ofribonucleic acid. Endocrinology 151, 1391-1397. doi: 10.1210/en.2009-1250

Kirkby, K. A., and Adin, C. A. (2006). Products of heme oxygenase and their potential therapeutic applications. Am. J. Physiol. Renal Physiol. 290, F563-F571. doi: 10.1152/ajprenal.00220.2005

Kitamuro, T., Takahashi, K., Ogawa, K., Udono-Fujimori, R., Takeda, K., Furuyama, K., et al. (2003). Bach1 functions as a hypoxia-inducible repressor for the heme oxygenase-1 gene in human cells. J. Biol. Chem. 278, 9125-9133. doi: 10. 1074/jbc.m209939200

Kuwano, Y., Rabinovic, A., Srikantan, S., Gorospe, M., and Demple, B. (2009). Analysis of nitric oxide-stabilized mRNAs in human fibroblasts reveals HuRdependent heme oxygenase 1 upregulation. Mol. Cell. Biol. 29, 2622-2635. doi: 10.1128/MCB.01495-08

Leautaud, V., and Demple, B. (2007). Regulation of heme oxygenase-1 mRNA deadenylation and turnover in NIH3T3 cells by nitrosative or alkylation stress. BMC Mol. Biol. 8:116. doi: 10.1186/1471-2199-8-116

Loya, C. M., Van Vactor, D., and Fulga, T. A. (2010). Understanding neuronal connectivity through the post-transcriptional toolkit. Genes Dev. 24, 625-635. doi: 10.1101/gad.1907710

Lu, X., Chen-Roetling, J., and Regan, R. F. (2014). Systemic hemin therapy attenuates blood-brain barrier disruption after intracerebral hemorrhage. Neurobiol. Dis. 70, 245-251. doi: 10.1016/j.nbd.2014.06.005

Maines, M. D. (1997). The heme oxygenase system: a regulator of second messenger gases. Annu. Rev. Pharmacol. Toxicol. 37, 517-554. doi: 10.1146/annurev. pharmtox.37.1.517

Milani, P., Amadio, M., Laforenza, U., Dell'Orco, M., Diamanti, L., Sardone, V., et al. (2013). Posttranscriptional regulation of SOD1 gene expression under oxidative stress: potential role of ELAV proteins in sporadic ALS. Neurobiol. Dis. 60, 51-60. doi: 10.1016/j.nbd.2013.08.005

Nakaso, K., Yano, H., Fukuhara, Y., Takeshima, T., Wada-Isoe, K., and Nakashima, K. (2003). PI3K is a key molecule in the Nrf2-mediated regulation of antioxidative proteins by hemin in human neuroblastoma cells. FEBS Lett. 546, 181-184. doi: 10.1016/s0014-5793(03)00517-9

Papadopoulou, C., Ganou, V., Patrinou-Georgoula, M., and Guialis, A. (2013). HuR-hnRNP interactions and the effect of cellular stress. Mol. Cell. Biochem. 372, 137-147. doi: 10.1007/s11010-012-1454-0

Pascale, A., Amadio, M., and Quattrone, A. (2008). Defining a neuron: neuronal ELAV proteins. Cell. Mol. Life Sci. 65, 128-140. doi: 10.1007/s00018-007-7017-y

Pascale, A., and Govoni, S. (2012). The complex world of post-transcriptional mechanisms: is their deregulation a common link for diseases? Focus on ELAVlike RNA-binding proteins. Cell. Mol. Life Sci. 69, 501-517. doi: 10.1007/s00018011-0810-7

Perrone-Bizzozero, N., and Bird, C. W. (2013). Role of HuD in nervous system function and pathology. Front. Biosci. (Schol. Ed.) 5, 554-563. doi: 10. $2741 /$ s389

Poon, H. F., Calabrese, V., Scapagnini, G., and Butterfield, D. A. (2004). Free radicals: key to brain aging and heme oxygenase as a cellular response to oxidative stress. J. Gerontol. A Biol. Sci. Med. Sci. 59, 478-493. doi: 10.1093/gerona/ 59.5.m478

Racchi, M., Mazzucchelli, M., Pascale, A., Sironi, M., and Govoni, S. (2003). Role of protein kinase $\mathrm{C}$ alpha in the regulated secretion of the amyloid precursor protein. Mol. Psychiatry 8, 209-216. doi: 10.1038/sj.mp.4001204

Romano, M., and Buratti, E. (2013). Targeting RNA binding proteins involved in neurodegeneration. J. Biomol. Screen. 18, 967-983. doi: 10. 1177/1087057113497256

Ryter, S. W., and Choi, A. M. (2002). Heme oxygenase-1: molecular mechanisms of gene expression in oxygen-related stress. Antioxid. Redox Signal. 4, 625-632. doi: 10.1089/15230860260220120

Scapagnini, G., Butterfield, D. A., Colombrita, C., Sultana, R., Pascale, A., and Calabrese, V. (2004). Ethyl ferulate, a lipophilic polyphenol, induces HO-1 and protects rat neurons against oxidative stress. Antioxid. Redox Signal. 6, 811-818. doi: $10.1089 / 1523086041798079$

Conflict of Interest Statement: The authors declare that the research was conducted in the absence of any commercial or financial relationships that could be construed as a potential conflict of interest.

Received: 20 July 2014; accepted: 17 December 2014; published online: 15 January 2015

Citation: Amadio M, Scapagnini G, Davinelli S, Calabrese V, Govoni S and Pascale A (2015) Involvement of ELAV RNA-binding proteins in the post-transcriptional regulation of HO-1. Front. Cell. Neurosci. 8:459. doi: 10.3389/fncel.2014.00459 This article was submitted to the journal Frontiers in Cellular Neuroscience.

Copyright (c) 2015 Amadio, Scapagnini, Davinelli, Calabrese, Govoni and Pascale. This is an open-access article distributed under the terms of the Creative Commons Attribution License (CC BY). The use, distribution and reproduction in other forums is permitted, provided the original author(s) or licensor are credited and that the original publication in this journal is cited, in accordance with accepted academic practice. No use, distribution or reproduction is permitted which does not comply with these terms. 